Article

\title{
An Infrared Actin Probe for Deep-Cell Electroporation-Based Single-Molecule Speckle (eSiMS) Microscopy
}

\author{
Sawako Yamashiro ${ }^{1, *}$ and Naoki Watanabe ${ }^{1,2}$ \\ 1 Laboratory of Single-Molecule Cell Biology, Kyoto University Graduate School of Biostudies, \\ Kyoto 606-8501, Japan; watanabe.naoki.4v@kyoto-u.ac.jp \\ 2 Department of Pharmacology, Kyoto University Graduate School of Medicine, Kyoto 606-8501, Japan \\ * Correspondence: yamashiro.sawako.5c@kyoto-u.ac.jp; Tel.: +81-75-753-4399
}

Received: 1 May 2017; Accepted: 22 June 2017; Published: 1 July 2017

\begin{abstract}
Single-molecule speckle (SiMS) microscopy is a powerful method to directly elucidate biochemical reactions in live cells. However, since the signal from an individual fluorophore is extremely faint, the observation area by epi-fluorescence microscopy is restricted to the thin cell periphery to reduce autofluorescence, or only molecules near the plasma membrane are visualized by total internal reflection fluorescence (TIRF) microscopy. Here, we introduce a new actin probe labeled with near infrared (NIR) emissive CF680R dye for easy-to-use, electroporation-based SiMS microscopy (eSiMS) for deep-cell observation. CF680R-labeled actin (CF680R-actin) incorporated into actin structures and showed excellent brightness and photostability suitable for single-molecule imaging. Importantly, the intensity of autofluorescence with respect to SiMS brightness was reduced to approximately 13\% compared to DyLight 550-labeled actin (DL550-actin). CF680R-actin enabled the monitoring of actin SiMS in actomyosin bundles associated with adherens junctions (AJs) located at 3.5-4 $\mu \mathrm{m}$ above the basal surfaces of epithelial monolayers. These favorable properties of CF680R-actin extend the application of eSiMS to actin turnover and flow analyses in deep cellular structures.
\end{abstract}

Keywords: single-molecule imaging; actin dynamics; near infrared probe

\section{Introduction}

Single-molecule fluorescence microscopy is one of the most sensitive detection techniques, and enables the visualization of individual molecules in cells. Live-cell single-molecule imaging has provided critical information on remodeling of the actin cytoskeleton [1-3], the motion of plasma membrane proteins [4,5], dynamics of microtubule motor proteins [6,7], and so on. The most accessible observation region for single-molecule imaging is the cell periphery and the area near the plasma membrane where background fluorescence can be reduced. On the other hand, there is a growing trend of extending the imaging approach to three-dimensional cellular structures by the development of new illumination systems. For example, highly inclined thin illumination (HILO) illuminates a thin section that is several microns deep in the sample [8]. The HILO technique has been used to observe targeting of chromatin regulatory complexes to chromatin [9], dynamics of transcription factors in the nucleus [10], and single-molecule dynamics near the cell cortex of Caenohabditis elegans embryos [11]. Gabhardt et al. developed reflected light-sheet microscopy, which enables selective plane illumination of a thin light sheet of $\sim 1 \mu \mathrm{m}$, and analyzed transcription factor-DNA interactions in the nucleus [12]. Lattice light-sheet microscopy achieves high image acquisition speed with frame rates more than 200 slices per second with a thin light-sheet illumination [13]. These selective plane imaging light-sheet microscopies for deep inside the cell are powerful technologies to monitor fast 
processes such as diffusion of molecules in the nucleus. On the other hand, various endogenous fluorophores including NADH, NADPH, flavins, lipofuscin-like lipopigments and vitamin A exist in the cytoplasm, which causes strong autofluorescence especially in the perinuclear region [14]. Furthermore, autofluorescence emission peaks of most endogenous fluorophores are 400-600 nm [14], which overlaps those of fluorophores employed for single-molecule imaging, such as EGFP $[4,6,11,15]$, tetramethylrhodamine [13], DyLight 549/550 [16,17], Dendra2 [10] and mEos2 [12]. Those issues of autofluorescence have hampered single-molecule imaging in the view field near the area emitting strong autofluorescence in the cytosplasm.

We recently developed a new, electroporation-based single-molecule speckle (eSiMS) microscopy [16,17] (also see our accompanying review). In this method, we employed an actin probe that is chemically labeled with a fluorescent DyLight 549 dye on lysine side chains. Electroporation-mediated delivery of DyLight 549-actin (DL549-actin) into cells enables the labelling of cells with $100 \%$ efficiency at the optimal low density suitable for SiMS microscopy. Using near infrared (NIR) emissive organic dyes extends eSiMS microscopy with fluorescence imaging in the NIR region where the background generated by cellular autofluorescence is low. Furthermore, NIR dyes are suitable for imaging in thick cells and tissues because fluorescence imaging in the long-wavelength region facilitates low phototoxicity to cells and deeper tissue penetration $[18,19]$. In this study, we introduce a new NIR actin labeled with CF680R dye for eSiMS microscopy and its application to single-molecule imaging of actin in cell-cell adhesion with epi-fluorescent microscopy.

\section{Materials and Methods}

\subsection{NIR-Labeled Actin}

Rabbit skeletal muscle actin labeled with DyLight649-NHS ester (Thermo Fisher Scientific: Waltham, MA, USA), CF680R-NHS ester (Biotium: Fremont, CA, USA), CF750-NHS ester (Biotium) and CF770-NHS ester (Biotium) were prepared as described previously [16,17].

\subsection{Plasmids}

The expression vector harboring the defective CMV promoter (delCMV) for Lifeact-mCherry was described previously [20]. The expression vectors for human vinculin tagged with EGFP at the N-terminus were described previously [16]. Human MRLC cDNA [21] was subcloned into a pTagRFP-T-C.

\subsection{Cell Culture and Electroporation}

XTC cells were maintained as described previously [15,22]. A6 cells were maintained as described previously [23]. XTC cells and A6 cells were subjected to electroporation to deliver NIR-labeled actin as described previously [16,17].

\subsection{Single-Molecule Speckle Imaging and Data Analysis}

SiMS imaging and live-cell imaging were carried out in XTC cells as described previously [17,22]. Briefly, XTC cells were allowed to spread on a poly-L-lysine-coated coverslip in 70\% L-15 Leibovitz medium (Invitrogen: Carlsbad, CA, USA) without serum. A6 cells were allowed to spread on a collagen (BD Biosciences: San Jose, CA, USA)-coated coverslip in 50\% L-15 Leibovitz medium without serum. To observe CF680R-actin in epithelial A6 monolayers, A6 cells were seeded at a density of $1 \times 10^{5}$ cells $/ \mathrm{cm}^{2}$ on a collagen-coated coverslip and maintained in 50\% L-15 Leibovitz medium with 10\% fetal bovine serum for $48 \mathrm{~h}$. Imaging was performed using a microscope (IX83, Olympus: Tokyo, Japan) equipped with $75 \mathrm{~W}$ xenon illumination and a cooled EMCCD camera (Evolve 512, Photometrics: Tucson, AZ, USA), or a microscope (IX71, Olympus) equipped with $100 \mathrm{~W}$ mercury illumination and a cooled EMCCD camera (Evolve 512; Photometrics). A PlanApo 1.40 NA 100× oil objective (Olympus) was used to observe spread cells on a coverslip, and a UPLSAPO60XS2 1.30 NA 
$60 \times$ silicone immersion objective (Olympus) was used to observe actin structures associated with adherens junctions (AJs). Speckles were analyzed by using Speckle TrackerJ as described $[16,17]$.

\section{Results}

To develop a NIR actin probe for eSiMS, we prepared actin labeled at lysines with DyLight 649, CF680R, CF750 and CF770 dyes, respectively. These actin probes were delivered into the cytoplasm of Xenopus XTC cells by the electroporation method [16,17]. We compared photostability of the actin probes in the cell peripheral region to exclude autofluorescence in the cell body. In XTC cells, CF680R-labeled actin (CF680R-actin) and DyLight 649-labeled actin (DL649-actin) were considerably photostable, whereas actin labeled with CF750 or CF770 dyes was photobleached immediately after the first image (Figure 1, Video S1). Brightness of CF680R-actin was similar to that of DL649-actin. In the following study, we employed CF680R-actin as a NIR actin probe for eSiMS, because CF680R-actin is excitable with longer wavelength than DL649-actin.

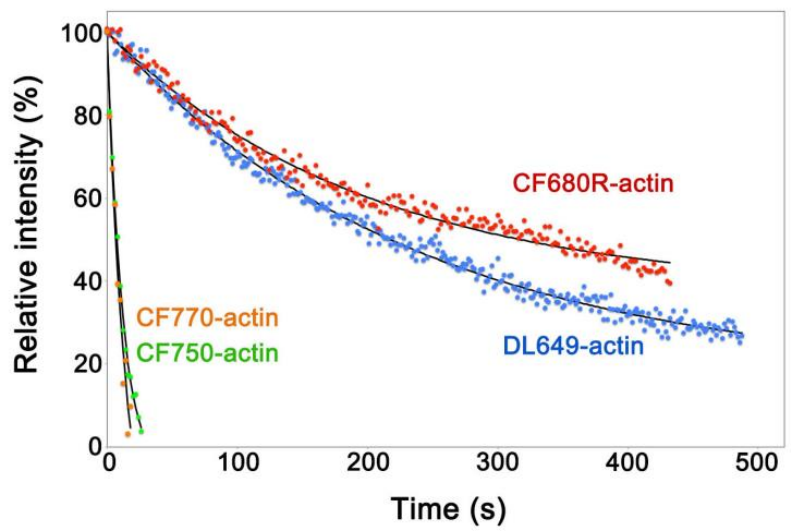

Figure 1. Intracellular photostability of fluorescent dye-labeled actin probes. XTC cells loaded with each fluorescent dye-labeled actin by electroporation were continuously exposed to excitation light using the $100 \mathrm{~W}$ mercury illumination system. The single-molecule speckle (SiMS) of each actin probe was acquired with $2 \mathrm{~s}$ exposure time without interval. The excitation light used was full for CF750- (green dots) and CF770-actin (orange dots), attenuated to 25\% for DL649-actin (blue dots) or $12 \%$ for CF680R-actin (red dots). The filter sets used were the Cy5 filter set (Semrock: Rochester, NY, USA) for DL649-actin, the Cy5.5-C filter set (Semrock) for CF680R-actin and the IRDYE 800 filter set (Semrock) for CF750- and CF770-actin. Black lines show the single-exponential curve fitted to the data. Relative intensity (y-axis) shows percentages derived from the fluorescence intensity of the peripheral region of the XTC cell divided by the value at the $y$-intercept of the fitted curve. The data shown are representative of at least two independent experiments.

We replaced the excitation filter (FF02-655/40-25, transmission wavelength 635-675 nm, Semrock) of the Cy5.5-C filter set (Semrock) for that with narrower transmission wavelength $651-671 \mathrm{~nm}$ (FF01-661/20-25, Semrock) to minimize autofluorescence. Both the $75 \mathrm{~W}$ xenon and the $100 \mathrm{~W}$ mercury illumination systems worked for SiMS imaging of CF680R-actin. The xenon illumination was 4- to 5-fold stronger in visualizing single-molecule CF680R-actin than the mercury illumination. To achieve visualization of CF680R-actin SiMS with a $1 \mathrm{~s}$ exposure time, the xenon excitation was attenuated to $6-12 \%$ using neutral density filters whereas the mercury excitation was attenuated to $25-50 \%$. Photobleaching of CF680R-actin was slow under these conditions. Continuous exposure of the whole cell area to $6 \%$ xenon excitation for $200 \mathrm{~s}, 400 \mathrm{~s}$ and $600 \mathrm{~s}$ reduced CF680R-actin fluorescence to $82.0 \%, 73.1 \%$ and $62.9 \%$, respectively (Figure 2A, Video S2). Therefore, CF680R-actin can be used to track actin SiMS for several minutes without a large influence of photobleaching. The brightness and photostability of CF680R-actin was comparable to those of DL549- or DL550-actin. The mercury illumination, which has a strong peak at the wavelength of around $546 \mathrm{~nm}$, is suitable for SiMS imaging 
of DL549-actin and can be attenuated to $1.5 \%$ to visualize DL549-actin speckles [16]. Continuous exposure of the whole cell area to $1.5 \%$ of the mercury excitation for $600 \mathrm{~s}$ reduces DL549-actin fluorescence to only $76 \%$ of the original value [16].
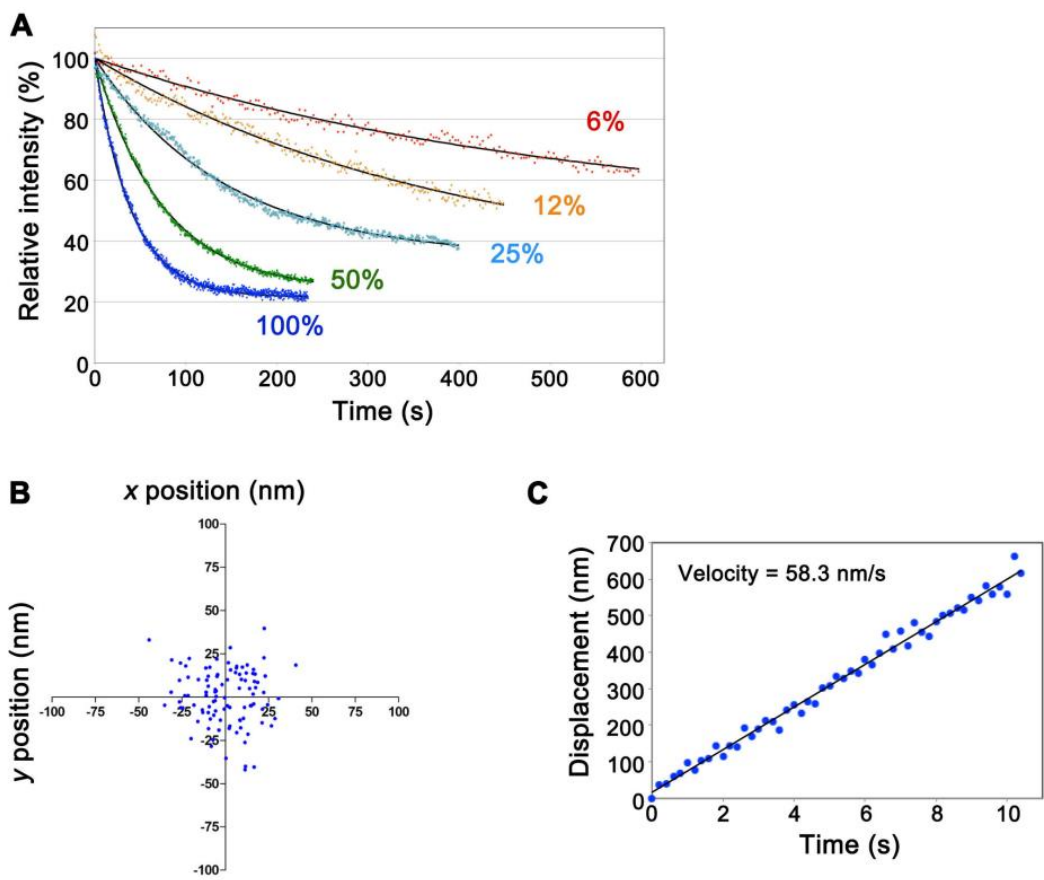

Figure 2. Characterization of CF680R-actin in live XTC cells. (A) Photostability of CF680R-actin using excitation light with the $75 \mathrm{~W}$ xenon illumination system in live cells. The excitation light used was full $(100 \%)$ or attenuated to $50 \%, 25 \%, 12 \%$ or $6 \%$ as indicated. Black lines show the single-exponential curve fitted to the data. The data shown are representative of three independent experiments; (B) Dispersion of the central position of the immobile glass-bound CF680R-actin speckle acquired with a $200 \mathrm{~ms}$ exposure time and a full $75 \mathrm{~W}$ xenon excitation (fast-tracking images, 100 frames). The standard deviations of the centroid positions in one direction are $16.3 \mathrm{~nm}$ for the $x$-axis and $16.0 \mathrm{~nm}$ for the $y$-axis. The data shown are representative of the data from three independent experiments; (C) Displacement plot of the central position of CF680R-actin in lamellipodia in the series of fast-tracking images (50 frames). Note that CF680R-actin speckles moved with the retrograde actin flow in lamellipodia. The velocity of the CF680R-actin speckles was calculated from a linear fit. The data shown are representative of the data from three independent experiments.

We next examined whether CF680R-actin enables nanometer-scale displacement measurement as we reported with DL549-actin previously [16]. In the nanometer-scale displacement analysis, DL549-actin achieves low localization error of 8-8.5 $\mathrm{nm}$ and measurement of the speed of the actin flow within 100-150 $\mathrm{nm}$ that is below the diffraction limit of the light microscope in live cells [16]. The unattenuated $75 \mathrm{~W}$ xenon illumination was used to monitor CF680R-actin speckles with a high signal-to-noise ratio. We acquired images at $200 \mathrm{~ms}$ intervals (fast tracking) for the short duration of $10 \mathrm{~s}$. Subpixel localization of the centroids of CF680R-actin SiMS was determined by using the two-dimensional Gaussian fit model of Speckle TrackerJ [16]. Under this condition, the centroids of immobile CF680R-actin SiMS that were immobilized on the glass surface were distributed with standard deviation of $16.3 \mathrm{~nm}$ and $16.0 \mathrm{~nm}$ in the $x$ - and $y$-axes, respectively (Figure 2B). CF680R-actin SiMS incorporated into the lamellipodial actin network moving along the retrograde actin flow as observed with EGFP-actin and DL549-actin $[15,16]$. CF680R-actin enabled the measurement of the retrograde flow velocity of actin SiMS that had a short lifetime less than $10 \mathrm{~s}$ (Figure 2C). In the analysis of CF680R-actin, displacement over the distance of $200-300 \mathrm{~nm}$ for 3-5 s was sufficient for reliable measurement of the actin SiMS velocity. 
CF680R-actin SiMS incorporated into the actin meshwork in lamellipodia (Figure 3A) as is the case with DL549-actin [16]. Next, we compared the fluorescence intensity ratio of autofluorescence and fluorescent actin in XTC cells and Xenopus A6 kidney epithelial cells. We acquired autofluorescence under the conditions identical to those used for CF680R-actin SiMS (see the legend of Figure 3A) and DL550-actin SiMS (see the legend of Figure 3B). The brightness of DL550-actin SiMS (Supplementary Figure S1) was similar to CF680R-actin SiMS (Figure 3A) under the acquisition conditions for each actin probes described in the legend of Figure 3A,B. In A6 cells, the fluorescent intensities of DL550-actin SiMS and CF680R-actin were $1155 \pm 246.1$ and $1001 \pm 221.1$ (mean \pm SD, arbitrary unit), respectively. In either XTC cells or A6 cells, autofluorescence in the cell body was 5- to 6-fold lower with the filter set for CF680R-actin than with the filter set for DL550-actin (Figure 3B). In A6 cells, the intensity ratio of autofluorescence and SiMS brightness with CF680R-actin was reduced to approximately $13 \%$ of that with DL550-actin.

A

CF680R-actin speckles XTC cell
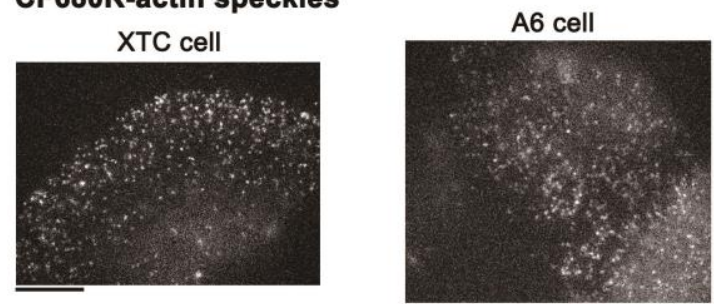

B
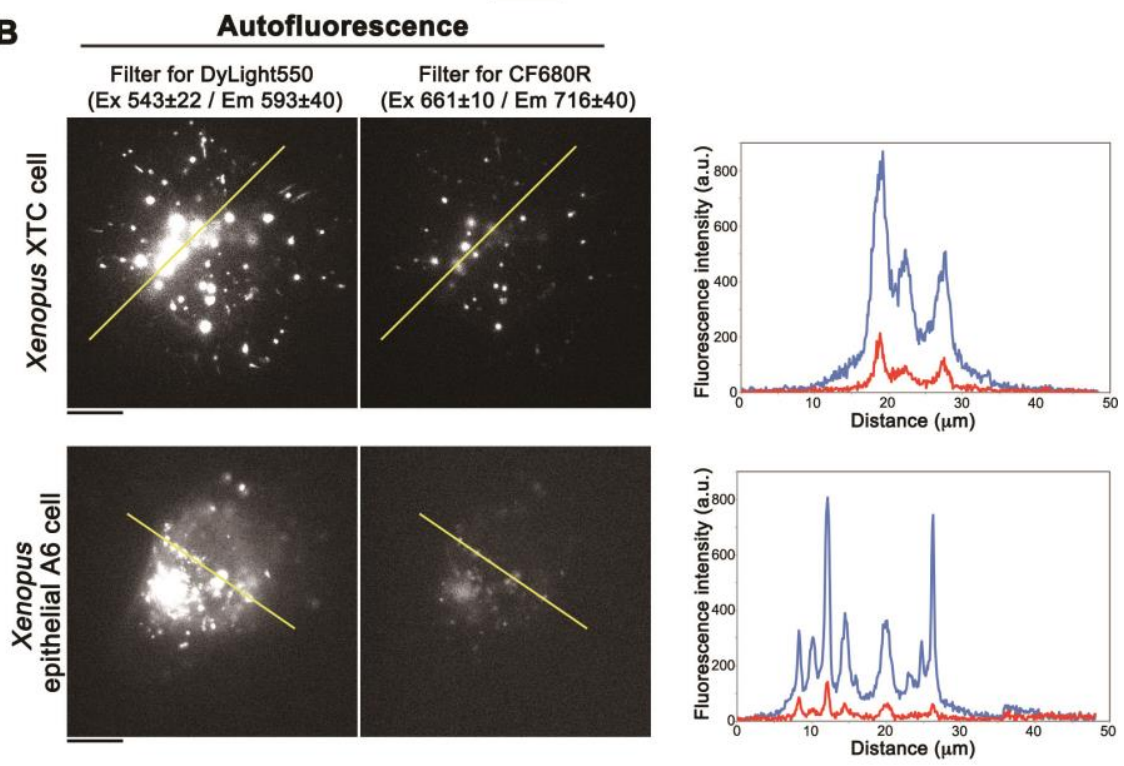

Figure 3. (A) Images of CF680R-actin SiMS in cell periphery of XTC cells (left) or A6 cells (right). The acquisition condition is with the custom filter set for CF680R-actin (an exciter filter: $661 \pm 10 \mathrm{~nm}$, an emitter filter: $716 \pm 40 \mathrm{~nm}$ and a dichroic beamsplitter: $685 \mathrm{~nm}$ ), the $75 \mathrm{~W}$ xenon illumination attenuated by a $12 \%$ neutral density filter, and a $1 \mathrm{~s}$ exposure time. Bar $=10 \mu \mathrm{m} ;(\mathbf{B})$ Autofluorescence images in an intact Xenopus XTC cell (upper) or A6 cell (lower) acquired under the conditions for DL550-actin SiMS (left) or CF680R-actin SiMS (right). The acquisition condition for DL550-actin SiMS is with the filter set for DL550-actin (Semrock TRITIC-B single-band filter set composed of an exciter filter: $543 \pm 20 \mathrm{~nm}$, an emitter filter: $593 \pm 40 \mathrm{~nm}$ and a dichroic beamsplitter: $562 \mathrm{~nm}$ ), the $75 \mathrm{~W}$ xenon illumination attenuated by a $12 \%$ neutral density filter and a $1 \mathrm{~s}$ exposure time. The acquisition condition for CF680R-actin SiMS is the same as in (A). The right graphs show fluorescence intensity measured by line-scan analysis along the yellow line in the images. Blue lines: Autofluorescence detected with the filter set for DL550. Red lines: Autofluorescence detected with the filter set for CF680R. Bar $=10 \mu \mathrm{m}$. The data shown are representative of four independent experiments. 
CF680R-actin with green and red fluorescent probes extends application of eSiMS microscopy to three-color imaging. At the cell-to-cell boundaries of XTC cells, CF680R-actin SiMS incorporated into actin bundles that were visualized with Lifeact-mCherry (Figure 4A). These actin bundles were originated from punctate adherens junctions marked with EGFP-vinculin (Figure 4A). In time-lapse images, CF680R-actin SiMS associating with the actin bundles moved toward the upper cell (yellow circles and arrow) or the lower cell (blue circles and arrow) at the cell-to-cell boundaries (Figure 4B). Interestingly, actin speckles above the punctate adherens junction in the images moved in two opposite directions: both upward and downward (Figure 4B). At sites of cell-cell contact, actin filaments are considered to be pulled toward the cell center by actomyosin contraction [24]. Our observation suggests coexistence of overlapping actin structures pulled in two distinct directions. Such actin structure might be related to adhesion zippers derived from filopodia of opposing cells [25]. Another possibility is that the actin speckles observed might have experienced a flow-like movement of actin at the tilted cell-cell junctions [26].

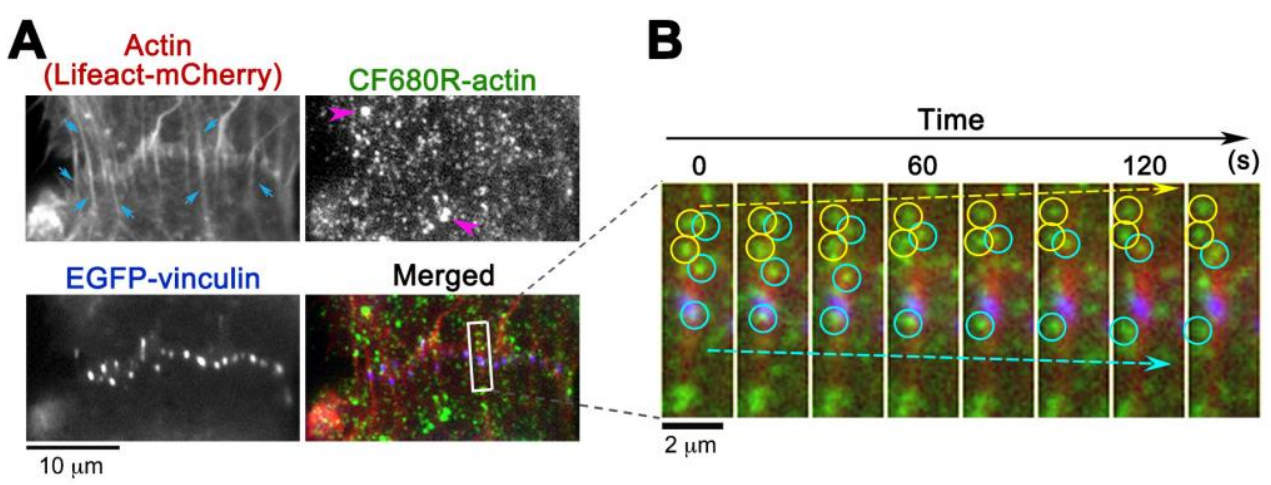

Figure 4. Observation of CF680R-actin SiMS in actin bundles at cell-to-cell boundaries of XTC cells. (A) CF680R-actin co-assembles to actin bundles (blue arrows) visualized by Lifeact-mCherry. The actin bundles are originated from punctate adherens junctions marked by EGFP-vinculin. Pink arrowheads in the CF680R-actin image indicate fluorescent organelle-like structures, which is apparently an artifact of electroporation; (B) Time-lapse images paneled at $20 \mathrm{~s}$ intervals in the area (rectangle) are shown on the right. Actin bundle-associated CF680R-actin SiMS moving upward (yellow arrow) and downward (blue arrow) are indicated by yellow (upward) or blue (downward) circles. Time-lapse images of CF680R-actin SiMS (green) are overlaid with the static images of Lifeact-mCherry (red) and EGFP-vinculin (blue).

CF680R-actin labeled bright organelle-like structures (Figure 4, pink arrows), as is the case with DL550-actin [16]. We speculate that the structure is an artifact arising from endocytosis of fluorescently labeled actin during electroporation [16,17]. Such organelle-like structures are markedly larger and brighter than SiMS and they move in stochastically changing directions [16]. We eliminated the structure from analysis.

The low background of CF680R-actin provides an opportunity to monitor actin structures in deeper cellular areas. In combination with epi-fluorescent microscopes, either SiMS using EGFP-tagged proteins or eSiMS employing DL550-actin has been mainly used to observe thin cell peripheral areas to avoid autofluorescence background. Using CF680R-actin, we visualized actin SiMS in actomyosin bundles associating with adherens junctions (AJs) of A6 cells forming epithelial monolayers with epi-fluorescent microscopy (Figure 5A,B, Video S3). In A6 cell monolayers, AJs are located at 3.5-4 $\mu \mathrm{m}$ from basal surfaces [23] and can be monitored within a single focal plane with a $60 \times$ silicone oil objective manufactured by Olympus. The silicone oil objective is suitable to observe deep cellular structures by reducing spherical aberration caused by refractive index (RI) mismatch. The RI of silicone oil for immersion medium is close to that of living tissues, which improves optical performance for imaging in thick samples. At the electron microscopic level, actin filament bundles running parallel 
to the plasma membrane have been observed in the zonula AJs [27]. CF680R-actin was presumably incorporated into such actin bundles. In the actin bundles, CF680R-actin SiMS stayed in almost the same position in a $10 \mathrm{~min}$ time window (Figure 5C). The average displacement of CF680R-actin SiMS for $10 \mathrm{~min}$ was $0.61 \pm 0.36 \mu \mathrm{m}$ ( $\mathrm{n}=19$ speckles). The slight movements of CF680R-actin SiMS in actin bundles appeared to accompany the changes in cell shape (Figure 5C).
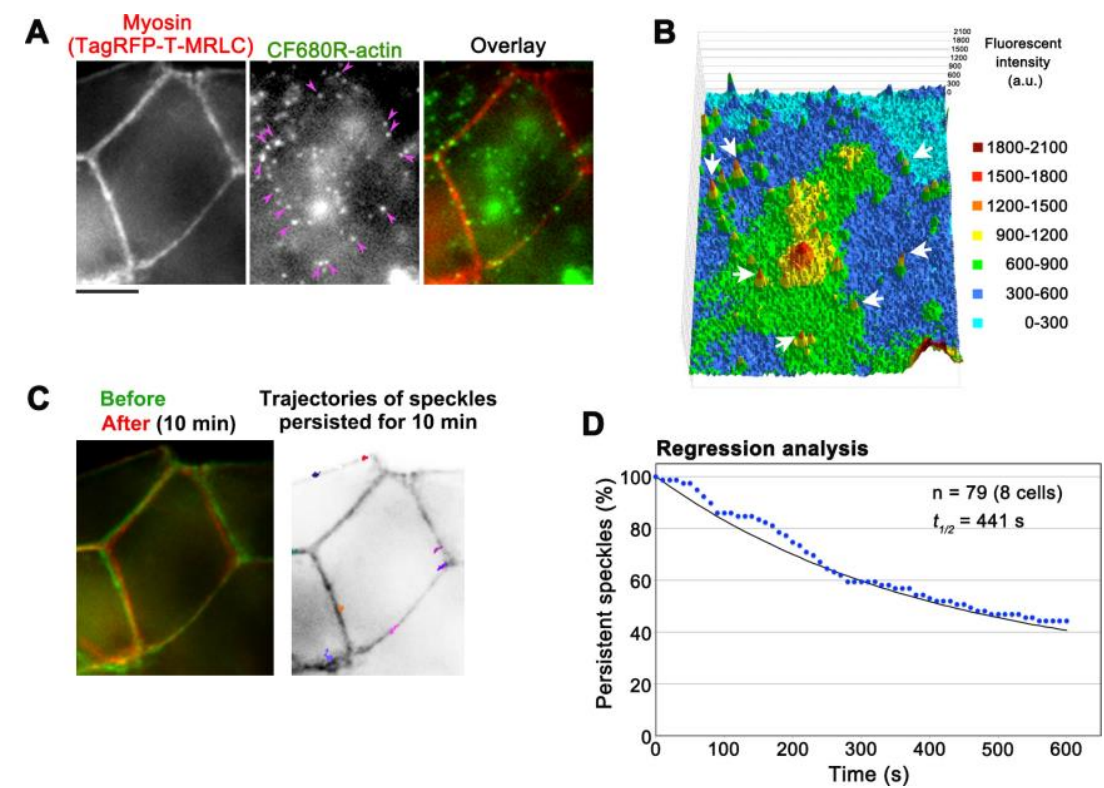

Figure 5. Observation of CF680R-actin SiMS in actomyosin bundles associated with adherens junctions (AJs) in A6 cells. (A) Representative images of actomyosin bundles visualized by TagRFP-T-myosin regulatory light chain (MRLC, left), CF680R-actin SiMS (middle), and the merged image (right). Pink arrowheads indicate CF680R-actin SiMS in actomyosin bundles associated with AJs. Bar $=5 \mu \mathrm{m}$; (B) The middle image of $(\mathbf{A})$ is shown in a 3D profile, in which the $z$-axis represents the fluorescence intensity of CF680R-actin. The fluorescence intensity of each pixel is shown with colors as indicated. White arrows indicate CF680R-actin SiMS; (C) Left: A merged image of actomyosin bundles associated with AJs visualized by TagRFP-T-MRLC before (green) and after (red) a $10 \mathrm{~min}$ time-lapse imaging of CF680R-SiMS. Right: Trajectories of CF680R-actin SiMS in actomyosin bundles associated with AJs. The trajectories of actin SiMS that persisted from the first image to the last image of a 10 min time-lapse imaging were indicated with colored lines and overlaid with the inverted image TagRFP-T-MRLC (before); (D) Speckle regression analysis of CF680R-actin SiMS in actomyosin bundles associated with AJs in eight cells. The black line indicates that the one-phase exponential curve fitted to the data (blue dots) gives $441 \mathrm{~s}$ for the half-life of SiMS.

We analyzed the disassembly rate of actin bundles by regression analysis of CF680R-actin SiMS, in which the surviving fraction of preexisting actin speckle is followed [15,22]. Our data revealed that actin filaments in actomyosin bundles associated with AJs disassembled with a half-life of $441 \mathrm{~s}$ (Figure 5D). As for actin structures in XTC cells, the average filament lifetime is $32.9 \mathrm{~s}$ in lamellipodia and $79.3 \mathrm{~s}$ in filopodia whereas the majority of actin filaments in actin stress fibers disassemble with a half-life of $5 \mathrm{~min}$ [16]. Therefore, the turnover of actin filaments in actomyosin bundles in AJs is relatively slow among actin structures observed in cultured cells.

One of the advantages of eSiMS for actin is that fluorescently labeled actin on lysine retains the ability to incorporate into actin filaments assembled by formin-homology proteins (formins) including mDia1 and mDia2 [16]. Formins are the major family of actin nucleators that also accelerate actin elongation [28-30], and play critical roles in the formation and stabilization of AJs formed in cultured cell systems and during Drosophila embryogenesis [31]. There are concerns about the usage of EGFP-actin for monitoring formin-based actin structures because several observations 
including our own indicated that EGFP-actin is not efficiently incorporated into actin filaments assembled by formins [16,32]. Therefore, the usage of EGFP-actin for the measurement of the actin turnover associated with AJs by fluorescence recovery after photobleaching (FRAP) in the previous studies [33,34] may have partially hindered monitoring of the formin-based actin turnover. In the present study, we were able to measure the turnover rate of actin filaments associated with AJs including formin-based actin networks for the first time.

\section{Conclusions}

In the present study, we introduced the new NIR emissive CF680R-actin probe for eSiMS microscopy. In live cells, single molecules of CF680R-actin can be visualized with low autofluorescence background, which is approximately $13 \%$ of eSiMS imaging with DL550-actin. In addition, CF680R-actin is considerably bright and photostable, which allows imaging of single molecules at high spatiotemporal resolution. These favorable properties of CF680R-actin would assist eSiMS imaging of deep cellular actin structures. Furthermore, CF680R-actin extends application of eSiMS microscopy to three-color single-molecule imaging. Its combination with green and red fluorescent probes will help researchers to visualize the molecular behavior of multiple cellular structures simultaneously in the same cell.

Supplementary Materials: The following are available online at http:/ /www.mdpi.com/1424-8220/17/7/1545/s1, Figure S1: Images of DL550-actin SiMS in cell periphery of XTC cell (left) or A6 cell (right) acquired under the acquisition condition for DL550-actin SiMS described in the legend of Figure 3B. Bar $=5 \mu \mathrm{m}$, Video S1: Intracellular photostability of fluorescent dye-labeled actin probes (Figure 1), Video S2: Photostability of CF680R-actin under attenuated xenon excitation (Figure 2A), Video S3: Time-lapse movie of SiMS of CF680R-actin in AJ of A6 cells (Figure 5A).

Acknowledgments: We thank Yuko Mimori-Kiyosue (Riken Institute) for providing us Xenopus laevis A6 cells and Hiroshi Hosoya (Hiroshima University) for pEGFP-MRLC. This work was supported by a Grant-in-Aid for Scientific Research on Innovative Areas Grant Number 00624347 from the Ministry of Education, Science, Sports and Culture of Japan (S.Y.), by JSPS KAKENHI Grant Number JP15K07045 (S.Y.), by JST-CREST Grant Number JPMJCR15G5 (N.W.) and by the Uehara Memorial Foundation (N.W.).

Author Contributions: S.Y. and N.W. conceived and designed the experiments; S.Y. performed the experiments; S.Y. analyzed the data; S.Y. and N.W. wrote the paper.

Conflicts of Interest: The authors declare no conflict of interest.

\section{References}

1. Watanabe, N. Inside view of cell locomotion through single-molecule: Fast F-/G-actin cycle and G-actin regulation of polymer restoration. Proc. Jpn. Acad. Ser. B Phys. Biol. Sci. 2010, 86, 62-83. [CrossRef] [PubMed]

2. Higashida, C.; Miyoshi, T.; Fujita, A.; Oceguera-Yanez, F.; Monypenny, J.; Andou, Y.; Narumiya, S.; Watanabe, N. Actin polymerization-driven molecular movement of mDia1 in living cells. Science 2004, 303, 2007-2010. [CrossRef] [PubMed]

3. Miyoshi, T.; Tsuji, T.; Higashida, C.; Hertzog, M.; Fujita, A.; Narumiya, S.; Scita, G.; Watanabe, N. Actin turnover-dependent fast dissociation of capping protein in the dendritic nucleation actin network: Evidence of frequent filament severing. J. Cell Biol. 2006, 175, 947-955. [CrossRef] [PubMed]

4. Douglass, A.D.; Vale, R.D. Single-molecule microscopy reveals plasma membrane microdomains created by protein-protein networks that exclude or trap signaling molecules in T cells. Cell 2005, 121, 937-950. [CrossRef] [PubMed]

5. Kasai, R.S.; Kusumi, A. Single-molecule imaging revealed dynamic GPCR dimerization. Curr. Opin. Cell Biol. 2014, 27, 78-86. [CrossRef] [PubMed]

6. Ananthanarayanan, V.; Schattat, M.; Vogel, S.K.; Krull, A.; Pavin, N.; Tolic-Norrelykke, I.M. Dynein motion switches from diffusive to directed upon cortical anchoring. Cell 2013, 153, 1526-1536. [CrossRef] [PubMed]

7. Cai, D.; McEwen, D.P.; Martens, J.R.; Meyhofer, E.; Verhey, K.J. Single molecule imaging reveals differences in microtubule track selection between Kinesin motors. PLoS Biol. 2009, 7, e1000216. [CrossRef] [PubMed]

8. Tokunaga, M.; Imamoto, N.; Sakata-Sogawa, K. Highly inclined thin illumination enables clear single-molecule imaging in cells. Nat. Methods 2008, 5, 159-161. [CrossRef] [PubMed] 
9. Zhen, C.Y.; Tatavosian, R.; Huynh, T.N.; Duc, H.N.; Das, R.; Kokotovic, M.; Grimm, J.B.; Lavis, L.D.; Lee, J.; Mejia, F.J.; et al. Live-cell single-molecule tracking reveals co-recognition of H3K27me3 and DNA targets polycomb Cbx7-PRC1 to chromatin. Elife 2016, 5, e17667. [CrossRef] [PubMed]

10. Izeddin, I.; Recamier, V.; Bosanac, L.; Cisse, I.I.; Boudarene, L.; Dugast-Darzacq, C.; Proux, F.; Benichou, O.; Voituriez, R.; Bensaude, O.; et al. Single-molecule tracking in live cells reveals distinct target-search strategies of transcription factors in the nucleus. Elife 2014, 3, e02230. [CrossRef] [PubMed]

11. Robin, F.B.; McFadden, W.M.; Yao, B.; Munro, E.M. Single-molecule analysis of cell surface dynamics in Caenorhabditis elegans embryos. Nat. Methods 2014, 11, 677-682. [CrossRef] [PubMed]

12. Gebhardt, J.C.; Suter, D.M.; Roy, R.; Zhao, Z.W.; Chapman, A.R.; Basu, S.; Maniatis, T.; Xie, X.S. Single-molecule imaging of transcription factor binding to DNA in live mammalian cells. Nat. Methods 2013, 10, 421-426. [CrossRef] [PubMed]

13. Chen, B.C.; Legant, W.R.; Wang, K.; Shao, L.; Milkie, D.E.; Davidson, M.W.; Janetopoulos, C.; Wu, X.S.; Hammer, J.A., 3rd; Liu, Z.; et al. Lattice light-sheet microscopy: Imaging molecules to embryos at high spatiotemporal resolution. Science 2014, 346, 1257998. [CrossRef] [PubMed]

14. Croce, A.C.; Bottiroli, G. Autofluorescence spectroscopy and imaging: A tool for biomedical research and diagnosis. Eur. J. Histochem. 2014, 58, 2461. [CrossRef] [PubMed]

15. Watanabe, N.; Mitchison, T.J. Single-molecule speckle analysis of actin filament turnover in lamellipodia. Science 2002, 295, 1083-1086. [CrossRef] [PubMed]

16. Yamashiro, S.; Mizuno, H.; Smith, M.B.; Ryan, G.L.; Kiuchi, T.; Vavylonis, D.; Watanabe, N. New single-molecule speckle microscopy reveals modification of the retrograde actin flow by focal adhesions at nanometer scales. Mol. Biol. Cell 2014, 25, 1010-1024. [CrossRef] [PubMed]

17. Yamashiro, S.; Mizuno, H.; Watanabe, N. An easy-to-use single-molecule speckle microscopy enabling nanometer-scale flow and wide-range lifetime measurement of cellular actin filaments. Methods Cell Biol. 2015, 125, 43-59. [PubMed]

18. Weissleder, R. A clearer vision for in vivo imaging. Nat. Biotechnol. 2001, 19, 316-317. [CrossRef] [PubMed]

19. Frangioni, J.V. In vivo near-infrared fluorescence imaging. Curr. Opin. Chem. Biol. 2003, 7, 626-634. [CrossRef] [PubMed]

20. Ryan, G.L.; Petroccia, H.M.; Watanabe, N.; Vavylonis, D. Excitable actin dynamics in lamellipodial protrusion and retraction. Biophys. J. 2012, 102, 1493-1502. [CrossRef] [PubMed]

21. Uchimura, T.; Fumoto, K.; Yamamoto, Y.; Ueda, K.; Hosoya, H. Spatial localization of mono-and diphosphorylated myosin II regulatory light chain at the leading edge of motile HeLa cells. Cell Struct. Funct. 2002, 27, 479-486. [CrossRef] [PubMed]

22. Watanabe, N. Fluorescence single-molecule imaging of actin turnover and regulatory mechanisms. Methods Enzymol. 2012, 505, 219-232. [PubMed]

23. Mimori-Kiyosue, Y.; Matsui, C.; Sasaki, H.; Tsukita, S. Adenomatous polyposis coli (APC) protein regulates epithelial cell migration and morphogenesis via PDZ domain-based interactions with plasma membranes. Genes Cells 2007, 12, 219-233. [CrossRef] [PubMed]

24. Maruthamuthu, V.; Aratyn-Schaus, Y.; Gardel, M.L. Conserved F-actin dynamics and force transmission at cell adhesion. Curr. Opin. Cell Biol. 2010, 22, 583-588. [CrossRef] [PubMed]

25. Vasioukhin, V.; Bauer, C.; Mei, Y.; Fuchs, E. Directed actin polymerization is the driving force for epithelial cell-cell adhesion. Cell 2000, 100, 209-219. [CrossRef]

26. Kametani, Y.; Takeichi, M. Basal-to-apical cadherin flow at cell junctions. Nat. Cell Biol. 2007, 9, 92-98. [CrossRef] [PubMed]

27. Yonemura, S. Cadherin-actin interactions at adherens junctions. Curr. Opin. Cell Biol. 2011, 23, 515-522. [CrossRef] [PubMed]

28. Breitsprecher, D.; Goode, B.L. Formins at a glance. J. Cell Sci. 2013, 126, 1-7. [CrossRef] [PubMed]

29. Campellone, K.G.; Welch, M.D. A nucleator arms race: Cellular control of actin assembly. Nat. Rev. Mol. Cell Biol. 2010, 11, 237-251. [CrossRef] [PubMed]

30. Paul, A.S.; Pollard, T.D. Review of the mechanism of processive actin filament elongation by formins. Cell Motil. Cytoskeleton 2009, 66, 606-617. [CrossRef] [PubMed]

31. Grikscheit, K.; Grosse, R. Formins at the Junction. Trends Biochem. Sci. 2016, 41, 148-159. [CrossRef] [PubMed] 
32. Chen, Q.; Nag, S.; Pollard, T.D. Formins filter modified actin subunits during processive elongation. J. Struct. Biol. 2012, 177, 32-39. [CrossRef] [PubMed]

33. Grikscheit, K.; Frank, T.; Wang, Y.; Grosse, R. Junctional actin assembly is mediated by Formin-like 2 downstream of Rac1. J. Cell Biol. 2015, 209, 367-376. [CrossRef] [PubMed]

34. Kovacs, E.M.; Verma, S.; Ali, R.G.; Ratheesh, A.; Hamilton, N.A.; Akhmanova, A.; Yap, A.S. N-WASP regulates the epithelial junctional actin cytoskeleton through a non-canonical post-nucleation pathway. Nat. Cell Biol. 2011, 13, 934-943. [CrossRef] [PubMed]

(C) 2017 by the authors. Licensee MDPI, Basel, Switzerland. This article is an open access article distributed under the terms and conditions of the Creative Commons Attribution (CC BY) license (http:/ / creativecommons.org/licenses/by/4.0/). 